\title{
SUR LE NOMBRE DE STADES NAUPLIENS CHEZ EUCYCLOPS SERRULATUS
}

\author{
par Françoise Lescher-Moutou $\mathbf{E}^{1}$.
}

\begin{abstract}
L'étude morphologique et biométrique du développement postembryonnaire d'Eucyclops serrulatus (Fischer) a permis de montrer la présence de six stades naupliens chez cette espèce. Six catégories différentes d'exuvies ont été recueillies dans les élevages.
\end{abstract}

\section{The number of naupliar intars in Eucyclops serrulatus.}

A morphological and biometrical study of the postembryonic development of Eucyclops serrulatus (Fischer) demonstrates that six naupliar instars are present for this species. Six different exoskeletal casts have been found in cultures under laboratory conditions.

Le développement postembryonnaire des Cyclopides a fait l'objet de nombreuses recherches depuis les premières données de Jurine (1820) et de Claus (1858) sur Macrocyclops fuscus (Jurine, 1820), M. albidus (Jurine, 1820), Eucyclops serrulatus (Fischer, 1851), Ectocyclops phaleratus (Koch, 1833) et Cyclops insignis Claus, 1857. Cependant, jusqu'en 1970, date à laquelle Elgmork et Langeland reconnaissent de façon irréfutable, grâce à l'examen des exuvies, l'existence de six stades naupliens chez Cyclops scutifer Sars, 1863, l'ensemble des résultats ne permettait pas de fixer avec certitude si le développement postembryonnaire des Eucyclopinae et des Cyclopinae comporte cinq ou six stades naupliens.

Ainsi, Dietrich (1915) à qui nous devons le premier développement complet de Cyclops strenuus Fischer, 1851, Manfredi (1923, 1925), Lucks (1926), Amelina (1927), Gelmini (1928), Gurney (1933), Rylov (1948), Ravera (1953), Damian-Georgescu (1963), McLaren (1964), Faheem Khan (1965), Auvray et Dussart (1966), Lesoher-Moutoué (1966) et Dussart (1969), décomptent cinq stades naupliens chez différentes espèces de Cyclopidae. En revanche six stades sont décrits par Walter (1922), Ziegelmayer (1925), Ewers

1. Laboratoire souterrain du C.N.R.S., 09410 Moulis, France. 
(1930), Borutzky (1949), Dukina (1956), Mazepova (1960), Elgmork (1965), Eppacher (1968) et Elgmork et Langeland (1970).

Dans un travail récent (Lescher-Moutoué, 1973) j'ai montré, grâce à des observations d'ordre morphologique et biométrique, que les espèces hypogées Speocyclops racovitzai (Chappuis, 1923) et $S p$. gallicus Chappuis et Kiefer, 1952, présentent bien six stades naupliens, alors que l'espèce hypogée Graeteriella unisetigera (Graeter, 1908) en possède cinq. C'est essentiellement le décompte des exuvies prélevées dans les élevages au fur et à mesure des exuviations (chaque individu étant isolé dès l'éclosion de l'œuf) qui m'a permis de fixer avec certitude le nombre de stades naupliens de ces espèces : $S p$. racovitzai et $S p$. gallicus ont fourni six catégories d'exuvies naupliennes et $G$. unisetigera cinq catégories seulement.

Le développement postembryonnaire de I'Eucyclopinae Eucyclops serrulatus, espèce Iroglophile, ayant fait l'objet de nombreux travaux conduisant à des résultats contradictoires quant au nombre de stades naupliens (Manfredi, 1923 ; Ziegelmayer, 1925 ; Dukina, 1956 ; Auvray et Dussart, 1966 ; Kiefer, 1973), j’ai réalisé un élevage de cette espèce.

Les récoltes dans la nature ont été effectuées dans des abreuvoirs (commune de Moulis, Ariège), à l'aide de filets en gaze à bluter dont la maille mesure $95 \mu$, et triées au laboratoire.

Les observations ont été menées selon les mêmes techniques que celles utilisées pour Speocyclops et Graeteriella : plusieurs femelles ovigères ont été placées isolément dans une série de cuvettes d'élevage ; chacune d'entre elles était transvasée dès qu'elle perdait ses sacs ovigères. Les nauplii, prélevés régulièrement dans chaque cuvette, étaient fixés à l'alcool à $70^{\circ}$, éclaircis à l'acide lactique puis montés entre lame et lamelle dans ce même milieu pour l'examen microscopique et les mesures. D'autres nauplii ont été isolés dans des cuvettes d'élevage; les exuvies retirées au fur et à mesure de leur apparition ont ensuite été reconnues au microscope.

\section{Description des stades naupliens d'Eucyclops serrulatus.}

Pour chacun des nauplii suivis en élevage, six catégories d'exuvies ont été recueillies : le développement postembryonnaire d'E. serrulatus comporte donc six stades naupliens (fig. 1 à 4) ainsi caractérisés :

Nauplius 1.

Corps ovoïde. Trois paires d'appendices. Les antennules (A1) sont formées de quatre segments. Les antennes (A2), avec coxa et basis (une épine masticatrice sur le coxa), sont biramées : l'exopodite présente une segmentation indistincte (article distal orné de 

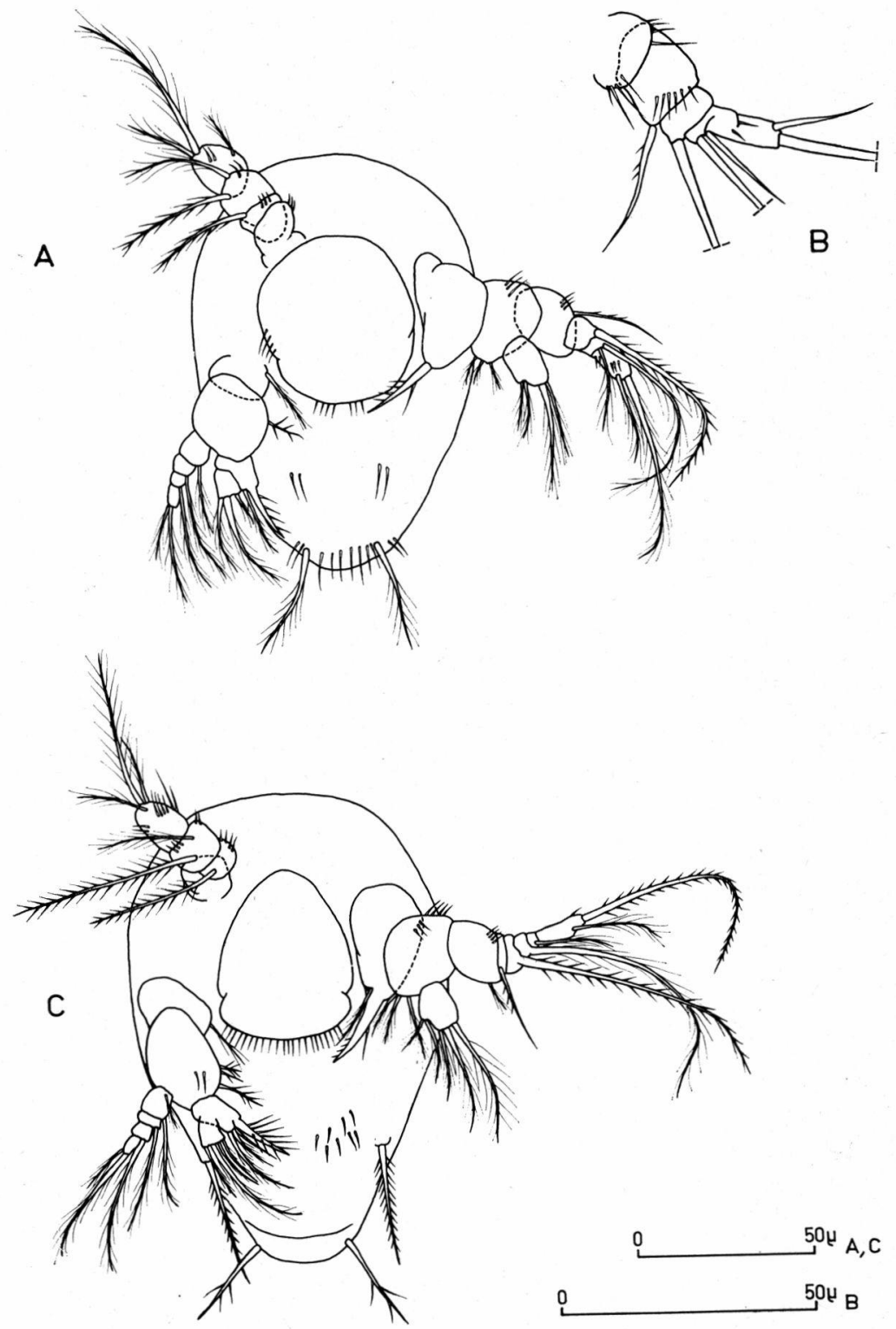

Fig. 1. - Fucyclops serrulatus. A : nauplius 1 (vue ventrale); B : détail de l'extıemité de l'exopodite de l'antenne du nauplius 1 ; $\mathrm{C}$ : nauplius 2 (vue ventrale)

deux soies) et l'endopodite porte quatre soies dont deux en position distale. Les mandibules (Md) sont également biramées ; le coxa et le basis supportent chacun une soie interne; l'exopodite est cons- 


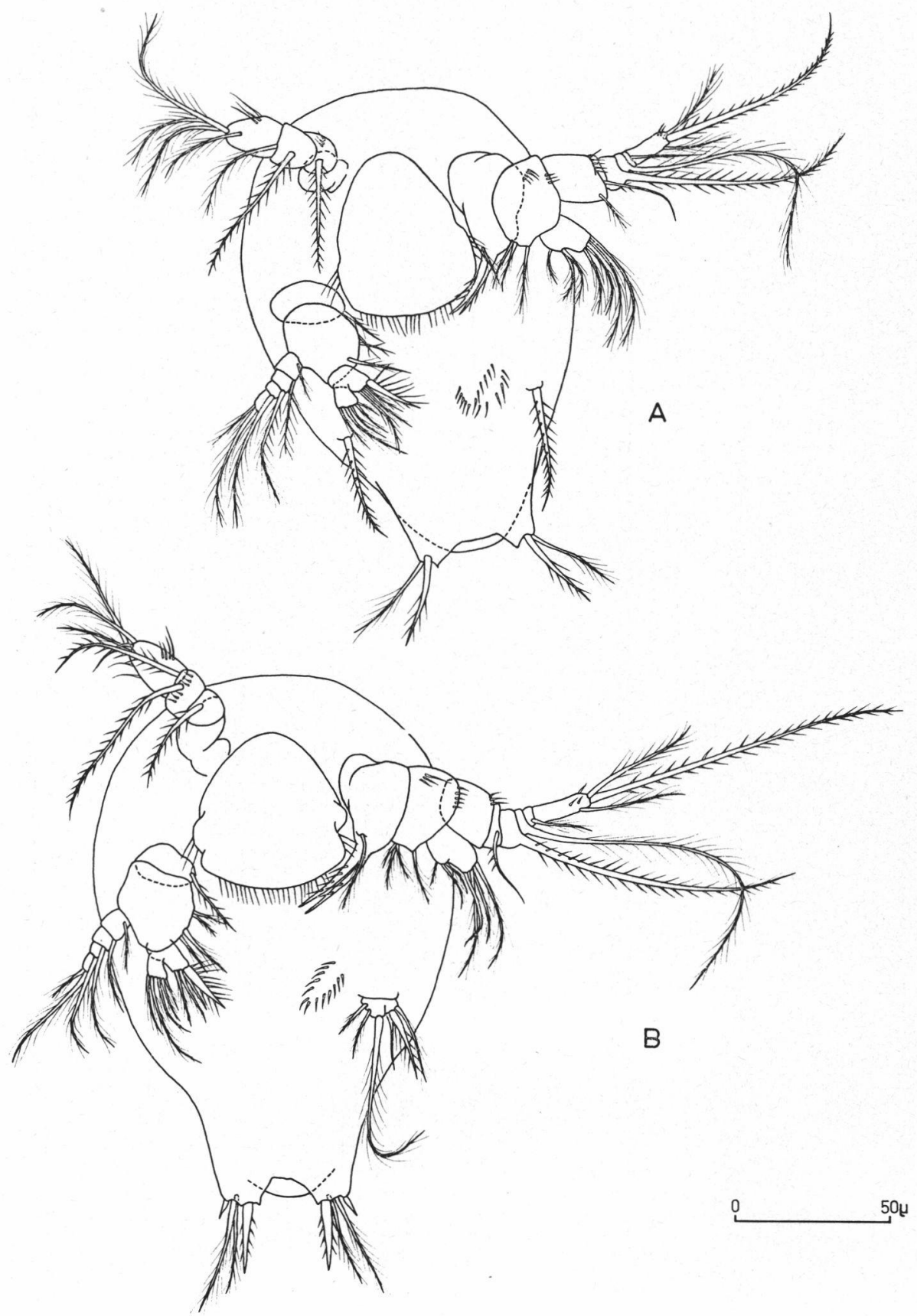

Fig. 2. - Eycyclops serrulatus. A : nauplius 3; B : nauplius 4 (vues ventrales). 
titué de quatre segments (deux soies sur l'article distal, une soie sur chacun des autres articles) cependant que l'endopodite, dont l'article basal porte une forte épine masticatrice interne et une fine soie, se prolonge par un article terminal orné de trois soies. Une seule paire de soies furcales finement ciliées.

\section{Nauplius 2.}

Corps ovoïde. Quatre paires d'appendices (A1, A2, Md, Mx1). L'ornementation des antennules est plus riche. Le coxa de l'antenne a une fine soie insérée à la base de l'épine masticatrice ; l'exopodite a six articles; l'endopodite est orné de cinq soies. L'endopodite des mandibules porte quatre soies insérées sur l'article distal et trois soies sur l'article basal en position interne par rapport à l'épine masticatrice; une soie ténue s'observe près de l'insertion de l'article basal de l'exopodite sur le basis. Les maxillules $(\mathrm{Mx} 1)$ sont représentées par une paire de soies densement barbelées. Une paire de soies furcales.

\section{NaUplius 3.}

Le corps, étiré vers l'arrière, présente une ébauche des branches furcales et quatre paires d'appendices (A1, A2, Md, Mx1). Il existe deux épines masticatrices sur le coxa de l'antenne outre la soie basale ; l'endopodite est orné de sept soies ; l'article distal de l'exopodite porte trois soies. L'article distal des mandibules est terminé par cinq soies. Deux paires de soies furcales sont présentes, la seconde paire vient s'insérer dorsalement par rapport à la première; toutes les soies sont finement ciliées.

\section{NAUPLIUS 4.}

Corps étiré. Quatre paires d'appendices (A1, A2, Md, Mx1). La maxillule est forméc de deux lobes peu différenciés ; de l'intérieur vers l'extérieur, le lobe interne porte deux courtes soies et une longue soie ciliée; le lobe externe s'orne d'une soie, d'une soie spiniforme et d'une seconde soie (six soies au total sur la maxillule, trois par lobe). De l'intérieur vers l'extérieur, trois paires d'épines et de soies furcales se répartissent ainsi : à une robuste épine barbelée font suite deux soies plumeuses, l'épine étant plus courte que les soies ; une courte expansion spiniforme externe apparaît également à ce stade.

\section{NaUplius 5.}

Corps, étiré en arrière de la région des maxillules. Quatre paires d'appendices (A1, A2, Md, Mx1). L'endopodite des antennes est orné de huit soies. Les maxillules sont nettement bilobées ; le lobe 


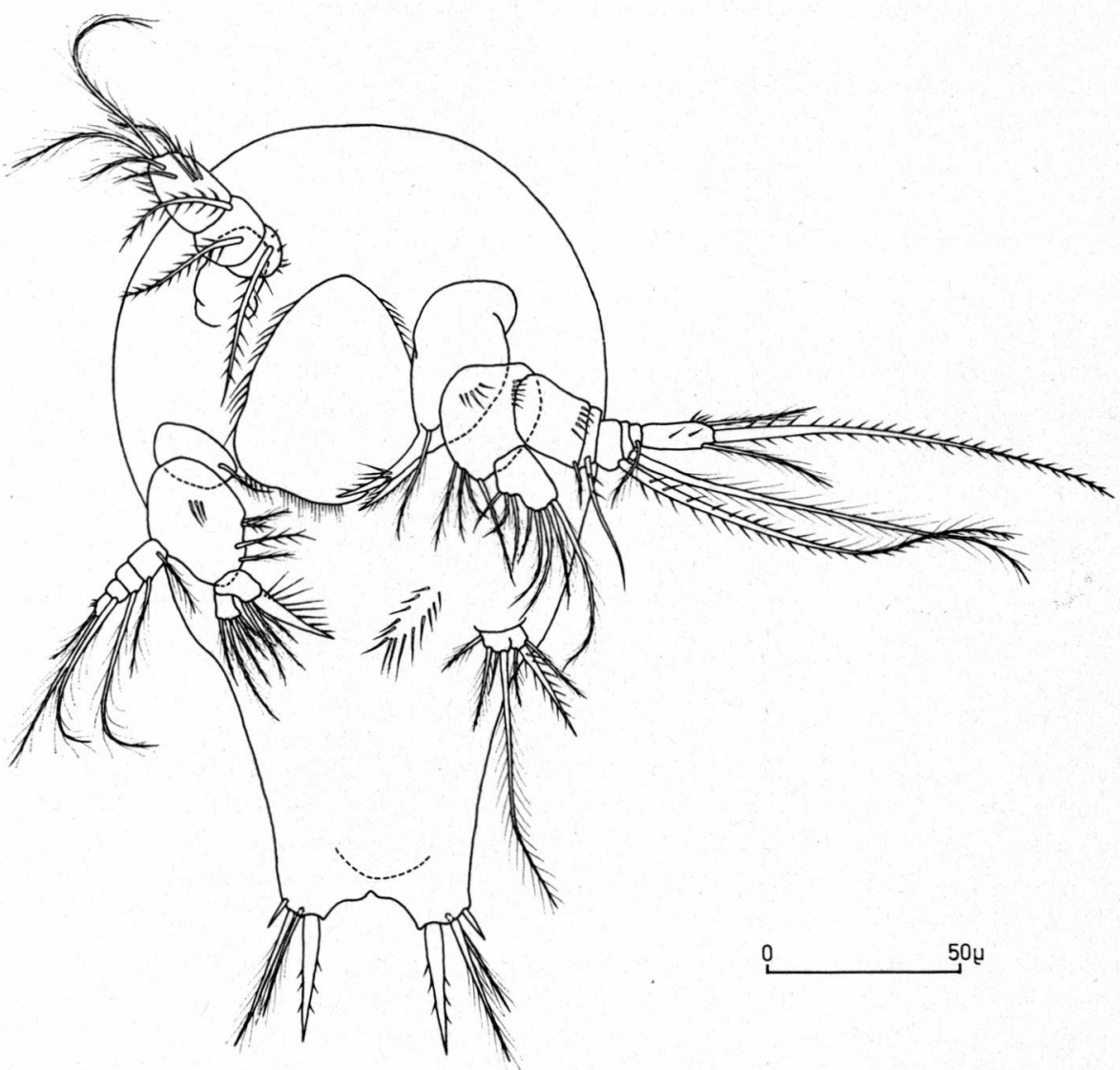

Fig. 3. - Eucyclops serrulatus. Nauplius 5 (vue ventrale).

interne porte trois courtes soies internes et une longue soie; le lobe externe possède une épine insérée entre deux soies (sept soies au total sur la maxillule, quatre sur le lobe interne et trois sur le lobe externe). L'ornementation furcale est identique à celle du nauplius 4 ; toutefois, l'épine interne présente une longueur comparable à celle des soies.

\section{Nauplius 6.}

Corps très étiré. Les appendices sont au nombre de sept paires du fait de l'apparition des ébauches des maxilles, des maxillipèdes et des deux premières paires de pattes thoraciques. L'endopodite 


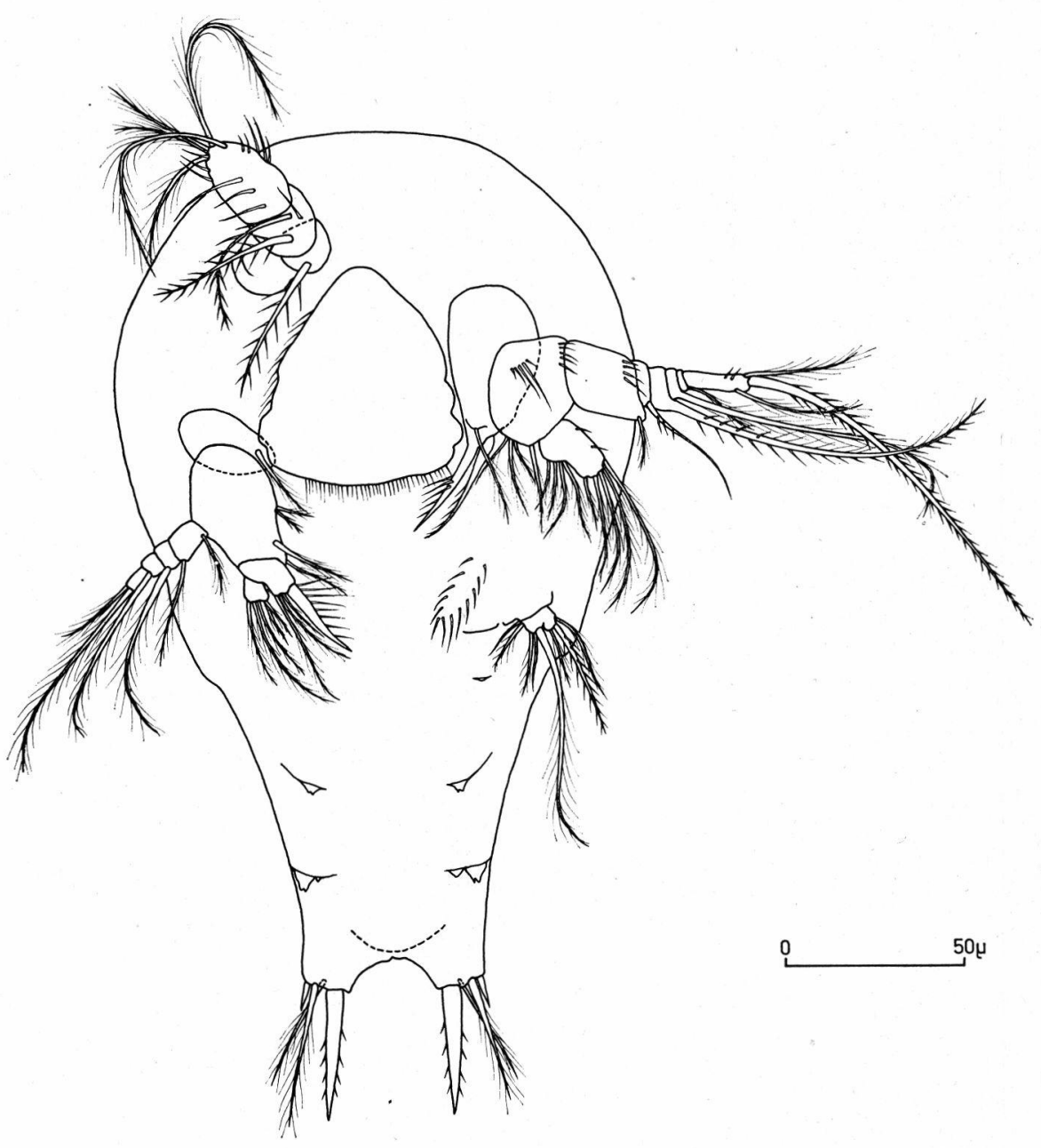

Fig. 4. - Eucyclops serrulatus. Nauplius 6 (vue ventrale).

des antennes est orné de neuf soies. Le lobe interne des maxillules supporte quatre courtes soies internes et une longue et forte soie ; le lobe externe est sans modifications (huit soies au total sur la maxillule). L'ornementation furcale n'off re aucun changement par rapport à celle des deux stades précédents.

Les trois derniers stades naupliens d'E. serrulatus montrent des caractéristiques suffisamment nettes pour qu'il soit aisé de les distinguer les uns des autres: 
- le nauplius 6 se reconnaît immédiatement par la présence de sept paires d'appendices ; le lobe interne de la maxillule s'orne de cinq soies ;

- les nauplii 4 et 5 possèdent la même armature furcale (outre les courtes expansions spiniformes externes, une paire d'épines internes et deux paires de soies plumeuses); cependant, l'épine est plus courte que les soies chez le nauplius 4 et sensiblement de la même longueur que celle-ci chez le nauplius 5 . Une différence apparaît également au niveau des maxillules : le lobe interne porte trois soies chez le nauplius 4 et quatre soies chez le nauplius 5 (fig. 5 ).
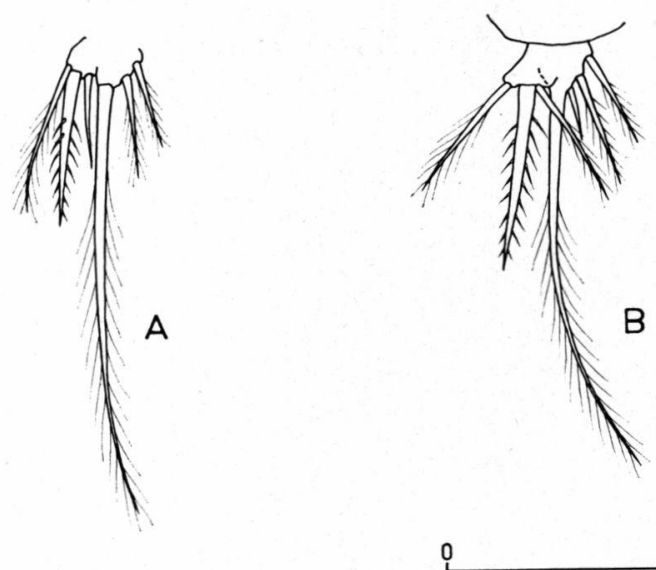

504

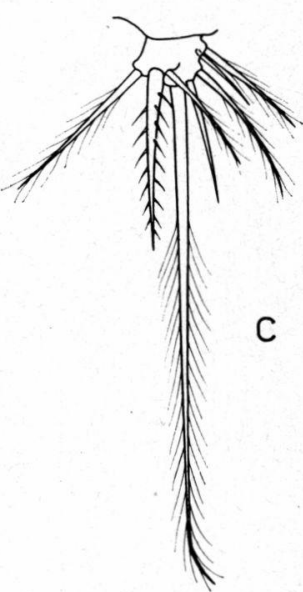

Fig. 5. - Maxillules du nauplius 4 (A), du nauplius 5 (B) et du nauplius 6 (C) d'Eucyclops serrulatus.

\section{Taille des stades naupliens d'Eucyclops serrulatus.}

Les mesures sur la longueur du corps de chaque stade nauplien sont regroupées dans le tableau I. Sont ensuite indiqués successivement la moyenne arithmétique $(\mathrm{X})$, le nombre de mesures (n) et l'erreur standard sur la moyenne (s) ; la longueur moyenne des différents stades naupliens est fournie au seuil de $5 \%$; enfin, un test de Student ( $t$ t) a été effectué.

Les valeurs moyennes de la taille de chaque stade nauplien s'accroissent régulièrement. Cependant, pour un stade donné, les valeurs maximales de longueurs sont supérieures aux valeurs mini- 
Tableau I. - Mesures de la longueur du corps des stades naupliens.

\begin{tabular}{|c|c|c|c|c|c|c|}
\hline Stade & N1 & N2 & N3 & $\mathrm{N} 4$ & N5 & N6 \\
\hline \multirow{9}{*}{ longueur } & 117,6 & 132 & 134 & 171,6 & 231 & 250 \\
\hline & 114 & 134,2 & 154 & 162,8 & 220 & 231 \\
\hline & 112,8 & 136,4 & 162,8 & 165 & 224,4 & 235,4 \\
\hline & 116,4 & 136,4 & 162,8 & 173,8 & 211 & 237,6 \\
\hline & 120 & 132 & 158,4 & 162,8 & 202,4 & 242 \\
\hline & 116,6 & 138,6 & 154 & 167,2 & 198 & 239,8 \\
\hline & 118,8 & 134,2 & 158,4 & 158,4 & 213,2 & 224,4 \\
\hline & 118,8 & 132 & 151,8 & 187 & 200,2 & 242 \\
\hline & 121 & 129,8 & 158,4 & 165 & 211 & 235,4 \\
\hline \multirow[t]{2}{*}{$d \mathbf{u}$} & 121 & 127,6 & 154 & 176 & 208,8 & \\
\hline & 107,8 & 121 & 158,6 & 171,6 & 200,2 & 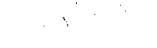 \\
\hline \multirow[t]{9}{*}{ corps : $\mu$} & 110 & 125,4 & 171,6 & 182,6 & 204,6 & \\
\hline & 110 & 123,2 & 147,4 & . & 211 & \\
\hline & 107,8 & 121 & 149,6 & & 217,6 & \\
\hline & 114,4 & 127,6 & 136,4 & & 217,6 & 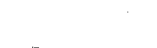 \\
\hline & 110 & 121 & 149,6 & & 193,6 & - \\
\hline & 114,4 & 123,2 & 154 & & & \\
\hline & 110 & 118,8 & 149,6 & & & \\
\hline & 110 & 116,6 & 151,8 & & & \\
\hline & 112,2 & & & & & \\
\hline $\mathrm{X}$ & 114,18 & 127,84 & 154,58 & 170,31 & 210,28 & 237,51 \\
\hline $\mathbf{n}$ & 20 & 19 & 19 & 12 & 16 & 9 \\
\hline $\mathbf{s}$ & 0,98 & 1,53 & 1,67 & 2,46 & 2,56 & 2,43 \\
\hline $\mathrm{L}_{\mu}$ & $114 \pm 2$ & $128 \pm 3$ & $154,5 \pm 3,5$ & $170 \pm 5,5$ & $210 \pm 5,5$ & $237,5 \pm 5,5$ \\
\hline$\ll t »$ & & & & 48 & 0,97 &, 02 \\
\hline
\end{tabular}

males du stade qui lui fait immédiatement suite (fig. 6). Remarquons également que, par sa longueur, le nauplius 4 est plus proche du nauplius 3 que du nauplius 5. Le test de Student permet en outre d'affirmer que les séries de mesures sont différentes au seuil de $1 \%$.

L'ensemble de ces résultats reposant sur des données d'ordre biométrique sont en accord avec les conclusions énoncées dans le paragraphe précédent : les six stades naupliens peuvent être parfaitement distingués chez Eucyclops serrulatus au cours d'une étude biométrique. 


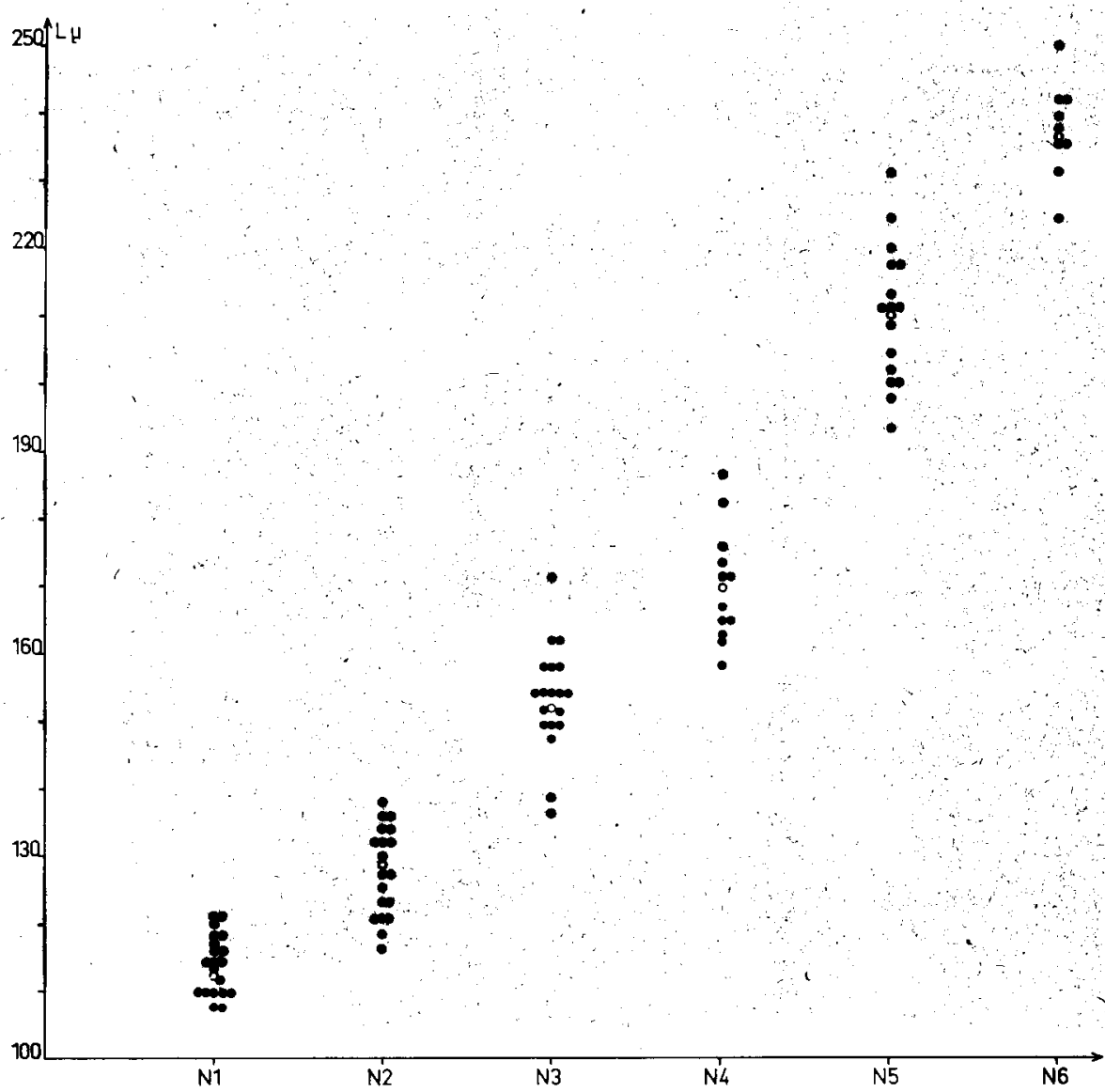

Fig. 6. - Longueur du corps des nauplii d'Eucyclops serrulatus aux différents stades (les cercles blancs représentent les valeurs moyennes).

\section{Conclusion.}

La reconnaissance de six exuvies naupliennes off rant des modifications morphologiques notables prouve l'existence de six stades naupliens au cours du développement postembryonnaire d'Eucyclops serrulatus. Ce résultat est confirmé par des données biométriques concernant la longueur du corps de chaque stade nauplien : elles permettent de distinguer six catégories de tailles; les différences entre leurs moyennes sont significatives.

Peut-être est-il possible dès à présent de postuler l'existence de six stades naupliens au cours du développement postembryonnaire pour la totalité du genre Eucyclops puisque, en outre, une observation identique a été effectuée à propos d'Eucyclops graeteri (Chappuis, 1927), espèce troglobie récemment maintenue en élevage au Laboratoire souterrain (Lescher-Moutoué, sous presse). 


\section{TRAVAUX CITÉS}

Amelina (L.). 1927. - Die Süsswasser Cyclopiden Iarva. Trudy kosin. biol. Sta., 6 : 31-39.

Auvray (C.) ef Dussart (B.). 1966. - Rôle de quelques facteurs du milieu sur le développement post-embryonnaire des Cyclopides (Crust. Cop.). I. Généralités, cas des Eucyclops. Bull. Soc. Zool. France, 91, $3: 477-491$.

Borutzky (E. V.). 1949. - Revue of the work of V. M. Rylov « Cyclopoida $\gg$ of freshwater. Fauna U.S.S.R., 3.

Claus (C.). 1858. - Zur anatomie und Entwicklungsgeschichte der Copepoden. Arch. Naturgesch., $24: 1-76$.

Damian-Georgescu (A.). 1963. - Crustacea Copepoda. Fam. Cyclopidae (forme de apa dulce). Fauna Rep. Pop. Romine, Ed. Acad. R.P.R., 4, $6: 1-205$.

Dietrich (W.). 1915. - Die Metamorphose der freilebenden Süsswasser Copepoden. I. Die Nauplien und das erste Copepodidstadium. Z. wiss. Zool., 113 : 252-324.

Dukina (V. V.). 1956. - Specific differences in the larvae of Cyclopidae. Zool. Zhurnal, 35 : 680-690.

Dussart (B.). 1969. - Les Copépodes des eaux continentales d'Europe occidentale (tome II). Cyclopoïdes et Biologie. Boubée, Paris : 1-292.

Elgmork (K.). 1965. - A triennal Copepod (Crustacea) in the temperate zone. Nature, London, $205: 413$.

Eigmork (K.) et LANGEland (A. L.). 1970. - The number of naupliar instars in Cyclopoida (Copepoda). Crustaceana, 18, 3 : 277-282.

Eppacher (T.). 1968. - Physiographie und zooplancton des Gossenköllesees. Ber. nat. med. Ver. Innsbruck, 56 : 31-123.

Ewers (L. A.). 1930. - The larval development of freshwater Copepoda. Contr. Frans Theodore Stone Inst. Hydrobiol., 3.

Faheem Khan (M.). 1965. - The effect of constant and varying temperatures on the development of Acanthocyclops viridis (Jurine). Proc. R. I. A., 64 (B), $8: 117-130$.

Germini (G.). 1928. - Contributo alla conoscenza dello sviluppo larvale di Cyclops leuckarti (Claus). Natura. Milano : 89-96.

Gurney (R.). 1933. - British freshwater Copepoda. Ray. Soc., London : 1-384.

JunrNe (L.) . 1820. - Histoire des Monocles qui se trouvent aux environs de Genève. Paris : 1-260.

Kiefer (F.). 1973. - Vergleichende Studien an Nauplien verschiedener Cyclopiden (Crustacea Copepoda). Mem. Ist. Ital. Idrobiol., 30 : 45-60.

Lescher-Moutoué (F.). 1966. - Note sur la reproduction et le développement postembryonnaire des Speocyclops. Ann. Spéléol., 21, $3: 673-687$.

Lescher-Moutoué (F.). 1973. - Sur la biologie et l'écologie des Copépodes Cyclopides hypogés (Crustacés). Ann. Spéléol., 28, 3 : 429502 et $4: 581-674$.

Lucks (R.). 1926. - Zur Entwicklung des Cyclops viridis Jur. und seiner Stellung zum Cyclops clausii. Heller. Schr. naturf. Ges. Danzig, 17 : 128-169. 
Manfredi (P.). 1923. - Etude sur le développement larvaire de quelques espèces du genre Cyclops. Ann. Biol. lac., 12 : 272-303.

Manfredi (P.). 1925. - Etude sur le développement larvaire de quelques espèces du genre Cyclops (2 note). Ann. Biol. lac., 14 : 111-129.

Mazepova (G. F.). 1960. - Morphologie des divers stades larvaires chez Cyclops kolensis (Cyclopoida) provenant du lac Baïkal. Izvest. Sibir Otdel Akad. Nauk. S.S.S.R., 6 : 103-115 (en russe).

McLaren (I. A.). 1964. - Zooplancton of lake Hazen, Ellsmere Island, and a nearly pond with special reference to the Copepod Cyclops scutifer Sars. Canad. Journ. Zool., 42 : 613-619.

Ravera (O.). 1953. - Gli stadi di sviluppo dei copepodi pelagici del lago Magiore. Mem. Ist. ital. Idrobiol., 7 : 129-151.

RYlov (V. M.). 1948. - Crustacea, Freshwater Cyclopoida. Fauna of U.S.S.R., $3,3: 1-314$.

Walter (E.). 1922. - Ueber die Lebensdauer der Freilebenden Süsswasser Cyclopiden und andere Fragen ihrer Biologie. Zool. Zh., $44: 375-420$.

Zielgelmayer (W.). 1925. - Metamorphose und Wachstum der Cyclopiden. Zeitschrift f. wiss. Zool., $126: 493-570$. 